$\mathbb{T}$ periodica polytechnica

Architecture

$39 / 2(2008) 59$

doi: 10.3311/pp.ar.2008-2.03

web: http://www.pp.bme.hu/ar

(c) Periodica Polytechnica 2008

RESEARCH ARTICLE

\section{Application of the techniques of traditional and modern fine arts in the architectural graphics}

\author{
Gábor Nemes
}

Received 2008-06-20

\begin{abstract}
The fundamental devices of the artistic representation. Possibilities of light / tone handling: xylographs, chiaroscuro, low tone differences, impressionistic tone handling. The use of colours in architectural graphics: emotional suggestivity, space suggestive role of the colours.

Pen/ink techniques: line drawings, brush and wash technique. The coloured drawings. Aquarelle. Pastel techniques. Coloured prints. Abstract plan compositions - the collage. Relation of architectural representation to modern abstract art.
\end{abstract}

\section{Keywords}

architectural graphics $\cdot$ pen $\cdot$ aquarelle $\cdot$ pastel $\cdot$ collage
Motto: "The drawing, named also as art of the draft, is the essence of the painting, sculpture and architecture."

(Michelangelo)

In the architect there always was and will be acclaim for the creation of plan graphics, presentations of independent value having aesthetic value, in addition to the technical plan documentation with its individual way of expression, characteristic to the architect and his architectural style. Plan graphics bearing the mark of the personality and unrepeatable individuality are expressing, beyond the technical content, already in the presentation projected into the plane, something about the spirituality of the planned building. Drawings act at the spiritual and also at an emotional level on the viewer, bringing the spectator nearer to acceptance of the architectural conception. If this striving is successful, then it can be safely stated that the design and sight plans may become equal associates to the artistic graphics by meeting the modern aesthetic criterion of projecting beyond itself.

Architectural graphics education just aims to familiarize and drill the students with the techniques and procedures which may promote the establishment of their independent graphic approach, choosing the one best suited to their architectural style i.e. their "messages" and individual habitudes. It does not require any special explanation that a faculty where education of the visual, aesthetic aspects of architecture is considered as the main profile, has to provide help to the architects to be, in shaping of their own graphic language, introducing and so to say offering the possible - existing - procedures so that the students then become able to start in the way most attractive for them, leading to the creation of graphics of artistic value. Another important goal of the education of this orientation is to increase the visual culture of the students - not feasible so efficiently by any other way- and to excite their interest for a more thorough cognition of the attendant arts.

The $20^{t h}$ century provided magnificent preliminaries for those dealing with architectural drawings of a graphic nature, here illustrated by a few examples:

Between the graphical and architectural graphical activities of Károly Kós, the great master of the secession opening the 
century, the boundaries are blurred; his tinted drawings recalling the superb, dramatic sombreness of the xylographs remind us of the creator essential in the wide sphere of visuality.

Pioneers of modernism, the De Stijl or the Bauhaus proclaimed the unity of the visual arts and simultaneously formulated the demand for fine-art determined architecture and architectural presentation of artistic rank, they created graphics exactly expressing the world of thought of the buildings.

The contemporary Hungarian architectural graphics may also attribute essential life-works as its own 1 in the thought-concise drafts of György Jánossy, sensitive drawings of György Vadász, inspired presentations of Imre Makovecz or just in the impressionalistically fresh aquarelles of lyrical intonation of Dr. István Balogh former Professor and Head of the Department of Design the borders are equally blurred between the artistic creations of sight plan initiation and those "pour l'art".

In a certain sense, architects of the present day have a more difficult task as compared to their historical predecessors since none of the architectural trends - likewise none of the fine-art trends - expresses entirely unambiguously the aesthetic standards. The various conceptual and visual worlds live in parallel equality naturally liberating the creative imagination but failing to give an exact basis for selection of the graphic representation adequate to the architectural thought. Only a thorough knowledge of the techniques and the modes of expression may help, as in Ariadne's yarn, it requires an orientation in the labyrinth of possibilities to find the Holy Grail of the authentic graphic expression at an artistic level, equal to that of its predecessors.

After this preamble, let us look at the devices and techniques concerned and familiarize ourselves with their details.

The fundamental devices of the artistic representation are: the line, the light tone and the colour ${ }^{2}$

In case of black-and-white representation the line and the tone are devices of artistic expression but at the same time the tone is also one of the fundamental elements of the colourful representation and thus it is natural that the tone - light-shadow values have a distinguished role in the representation.

\section{Possibilities for handling light / tone}

The drawing can be imagined with application of only black and white tones: this recalls the world of the xylographs. Here the black and white are not devices of the spatial expression, but they have a decorative role and function, acting on the emotions. By this means it is an artistic procedure suitable for the creation of expressive and elegant graphics of intense messages and decorative appearance.

Another peculiar possibility of the powerful light and tone handling is the chiaroscuro technique, giving a mysterious light

\footnotetext{
${ }^{1}$ Researching contemporary Hungarian architectural graphics is the specialist field of the author's $\mathrm{PhD}$ research.

${ }^{2}$ The author naturally does not forget the fundemental importance of the composition, but the teaching of this is an important point of origin for all subjects of the architects' education correlating with the design and visuality.
}

effect to the picture. Its essence is the concentration of the light on the image surface, which is suitable for the establishing of dramatic, sometimes mystic effects, capturing the viewer, making it to part of the theme, and leading to the world of the composition. Users of this technique improved their skills by painting often in candlelight, whose illumination brings the object to an entirely new tonal position, helping in the selection of the essential elements. The chiaroscuro effect can be observed on the creations illustrated. The drawings of high tonal difference feature dynamic and powerful effects.

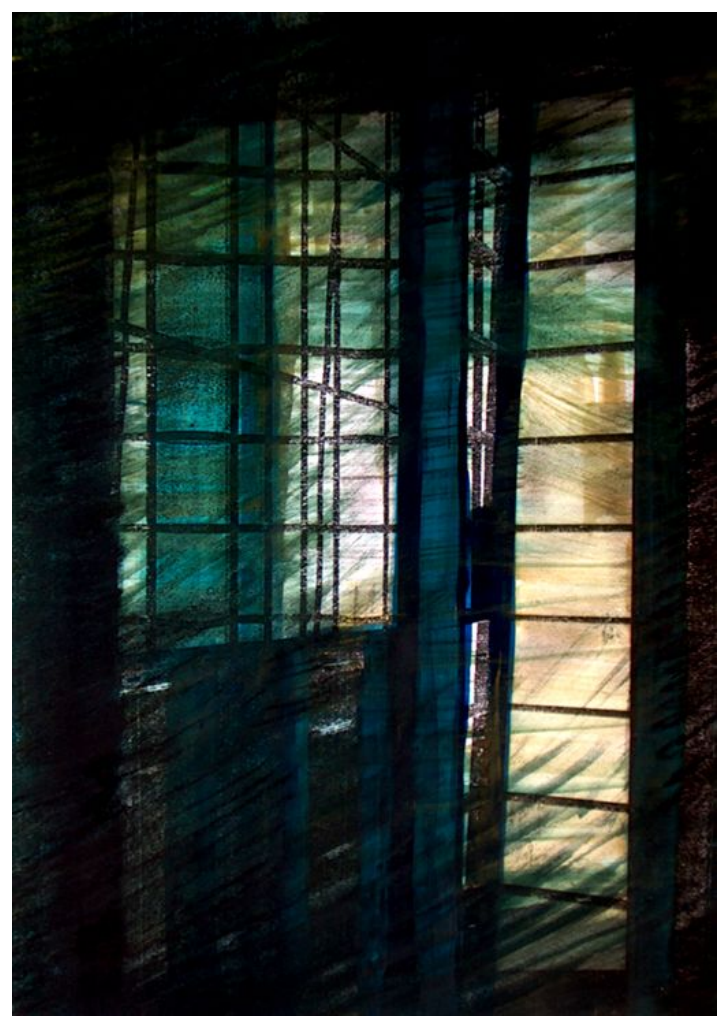

Fig. 1. Info building, student work (E. Szobota)

In case of drawings created with balanced tone conditions where there is a great distance between the light and dark tones but the composition is amended by rich intermediate tonal values - rational, elegant but slightly dull graphics are obtained.

The "pale" compositions of low tonal difference can be interesting, where just the lack of the tonal dynamism awakens the viewer's interest and the value of these creations is given instead of the impetus, by fine aesthetic, lyrical or dramatic "emptiness". Naturally in this "tonal greyness" many finely tuned tonal values participate and each picture element of striking lightness or darkness may have an especially high accent. If the tonal value of the picture is darker, then a picture of more gloomy and dramatic effect is obtained whilst if the tone is lighter, then the effect is naturally more cheerful and facile but the lack of the contrast remains in both cases sensible and makes the viewer an emotional part of the image world, which is an essential tool for the creator - let the composition be of fine art or architectural graphical incentive - in the transfer of the message. In poor light contrast, the dominating effects can be readily observed in 
the illustrated examples, where in one of the pictures there is a complete lack of the light, whilst in the other the light floods everything.

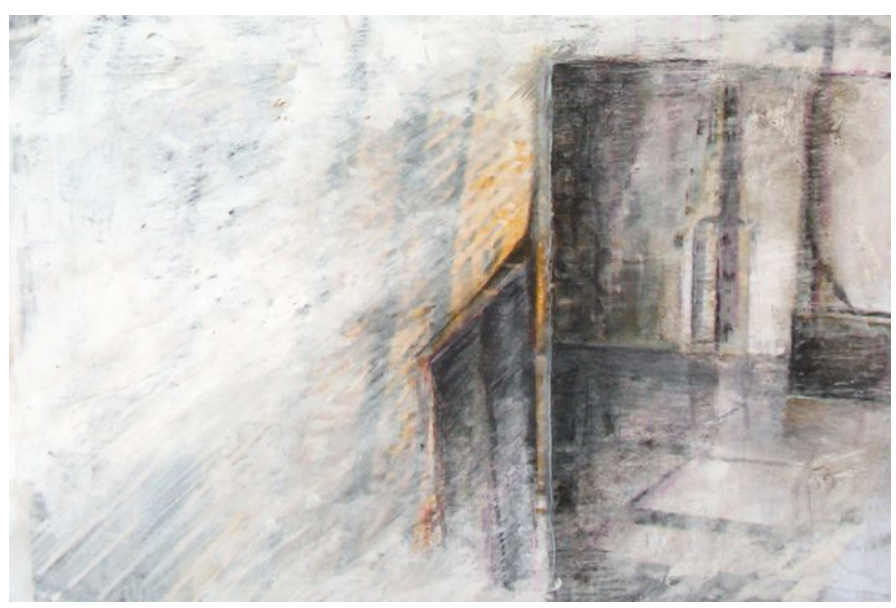

Fig. 2. Info building, student work (A. Hajas)

A similarly important possibility of handling the image's light - opposite to the chiaroscuro - is the creation of vibrating impressionistic - light effects that magically transforms the image and its theme, both facile and playful, causing the viewer to readily become a fan. Such airy and picturesque handling of the light is naturally one of the big achievements of French impressionism and, among others, this makes the architectural aquarelles of István Balogh so attractive.

\section{The Colour}

An even more rich possibility of the representation is inherent in utilizing the suggestiveness of colours. Colour and tone are naturally coherent notions, thus the above applies also to the colourful representation. Usage of colour at the same time can make the architectural graphic compositions even more pleasurably expressive. Naturally to achieve this, one also has to be familiar with the fundamental regularities of the colour contrasts as with the emotional and spatial suggestivity of the colours.

It is of general validity that powerful and expressive compositions are obtained with the utilization of so-called complementary colour pairs, where the dominant colours existing in the composition are located opposite to each other on the colour wheel. Conscious usage of the complementary colours is one of the definitive characteristics of the painting since impressionism. The more intensive, saturated colours present in the composition the more intensive stress is achieved.

Similarly dramatic effect is exerted also by the usage of the so-called cold-soft contrast, where the contrast is constituted by the blue and red colours.

Very great significance is attributed in the architectural graphics to the space suggestive role of the colours. The saturated, soft colours appear optically nearer than the non-saturated resp. the cold ones. Thus in a composition they "jump out" from the picture plane, becoming more accented whereby proper usage of the colours may provide a help in correct interpretation of the spatial conditions.

Conscious usage of the emotional suggestivity of the colours is also an important factor of the graphical composition. Here it is worth citing Goethe who in his colour theorem writes the following about the emotional effects of the colours: "Yellow: this colour is nearest to the light and it features a certain kind of sunny, cheerful and slightly stimulating property; the warmth and pleasure of the eyes can be attributed to the rubescent yellow; the red equally exerts the impression of earnestness and dignity as that of charm and grace. Lilac: features a certain vivacity without being jolly, more perturbing than exhilarating. The blue stimulates the feeling of cold, likewise it resembles the shadows. In its sight somehow the contradiction of the stimulus and the tranquillity is combined. Green: our eyes find a realistic satisfaction in this colour.

Accordingly, to achieve the requested emotional effect one may choose the basic colour mood of the graphics, then the further colours present in the composition and their superficial proportions can be selected to suit them".

And now, let us examine the various graphic techniques and the peculiarities of the visual effects that can be achieved with them.

\section{Black and White Techniques}

Black and white are sufficient to create a masterpiece. (Degas)

With the most direct and simplest of the drawing techniques - the pencil and the expressive possibilities thereof - the students are already thoroughly familiarized during the first few semesters, and thus the discussion of this technique will be ignored in this case.

Indian Ink (pen) techniques

The Indian ink pen respectively Indian ink as a paint material facilitates the creation of very versatile compositions of high suggestivity.

Indian Ink, as a Line Drawing

From the discussion of the above mentioned triumvirate line, tone, colour - not by chance is placed here the description of the possibilities offered by the line drawing, since the so-called "split" Indian ink pen hides rich possibilities of formulation in lines, as this device is pressure sensitive and thus it results in thicker lines as it is pressed more strongly to the paper.

The line is a result of an intensive abstraction since in nature only spatial forms exist and their sublimation into lines is created by the activity of the human brain. Accordingly, the line drawing is suitable for production of very sensitive, intellectually saturated works of art. The already mentioned variation of the line thickness only further enhances the suggestivity of this technique.

These so-called "single-line or linear" graphics facilitate the creation of very puritan and rational but at the same time emotionally heated drawings, capturing the essence of the reality either "the underlying reality" or representation focusing on this 
essence. These are very informative and concentrated drawings where minimum usage of the expressive devices nevertheless results in maximum intensity. Let us check these statements with the drawings of Béla Kondor and Lajos Varga.

The line drawing has another, more dynamic version, where from the tanglesome mixture of lines thrown vigorously to paper the form to be depicted makes itself felt.

Brush Drawing with Indian Ink, the Ink and Wash Technique

A branch of Indian ink drawing approaching to painting is the so-called washed Indian ink, where connection between the individual forms is established by picturesque, spot-like tones. Here the Indian ink is partly a line drawing device (suitable for creation of more filled, "sensual" lines with the use of a brush), and partly it is a medium diluted with water similar to the aquarelle, to be used in a similar way. By the contradiction of soft, mysterious transitions and the exactly formulated forms it can make the compositions created with the use of this technique very rich.

According to the intention of the creative expression the gauntness of the line drawing or placing the picturesque effects to the foreground can equally be a dominant feature of the graphics.

\section{The Charcoal / Chalk Drawing}

The charcoal respectively black, brown and red chalks offer wide-scale possibilities to the creator's presentation mode. Picturesque line drawings, compositions of dramatic tonal relations, or drawings of "breath like" fineness operating with circumstantial tonal differences can be created alike.

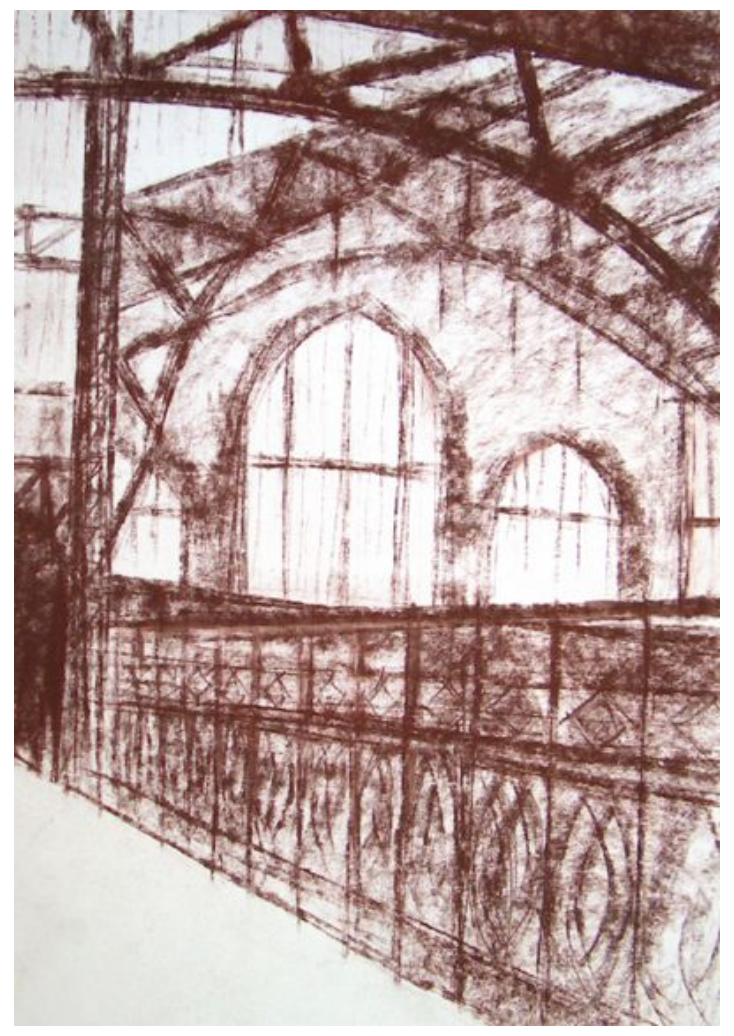

Fig. 3. Market hall, student work (N. Hajdu)

Anyhow, rather coordinated drawings can be created with the

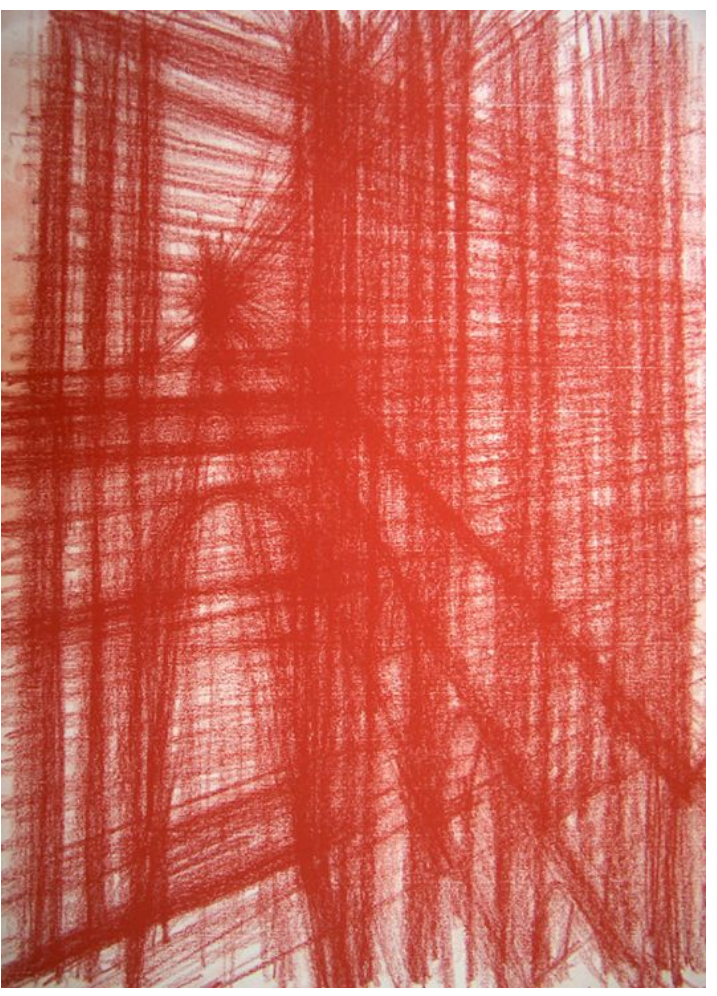

Fig. 4. Interior study, student work (A. Schreck)

application of this medium, which can be very helpful primarily in architectural graphics, in the creation of drafts and in quick illustrations of the mass conditions.

The charcoal drawing was, for example, a favoured sight plan drafting device of the early, expressive age of L. Mies van der Robe.

Peculiar, interesting and beautiful combinations of the application of the Indian ink drawing and charcoal can be imagined, the compositions of which simultaneously bear the characteristics of the gauntness of the line drawing and the soft picturesque of the charcoal.

\section{Colour Techniques}

\subsection{Aquarelle}

One of the most popular devices of the graphic, thus that of architectural graphics, a painting procedure created with the use of water soluble paints, built from transparent (glazing) layers.

Art of the "moment", whose essence is the rapid representation coming straight to the point, bearing the characteristics of the creator's impetus and freshness which then becomes one of the most important features of the beautiful aquarelles.

The aquarelle paint is a transparent and not densely covering paint and thus on the layers placed on one another the colours of the lower layers and even the white base colour of the paper are translucent, rendering these paintings alive with a peculiar light. It is expedient to solve the required accurate details with the device of the line drawing thus avoiding humdrum effects caused by possibly excessive accuracy of the painting, in contrast to the grandiose, energetic painted areas.

The aquarelle technique accompanied almost the entire his- 
tory of the painting. Its master was the British artist Turner, who in the $19^{\text {th }}$ century exploited to the greatest extent the light and atmosphere displaying capabilities of the aquarelle technique.

In turn in the $20^{t h}$ century, Paul Klee of Switzerland - a teacher at Bauhaus - splendidly expressed the atmospheres of the various lands, ages and music in his aquarelles.

From the cited masters much was learned by the perhaps greatest Hungarian artist of the architectural aquarelle, the already mentioned István Balogh. Let us observe the lightness of his pictures, the play of the light, the richness of the colours which, however, are always subordinated to the dominance of the ruling colour domain.

\subsection{The Pastel}

The pastel - and primarily the powder pastel - is a direct drawing device; its usage is similar to that of charcoal. By means of this technique, graphics of very versatile colour and of an emotional world can be created. The scale ranges widely from pictures of a dark, gloomy mood, to those of a fresh, healthy, light-infiltrated atmosphere.

An effect similar to that of the aquarelle can be achieved by washing - water-diluted painting - off the powder pastel.

Of the great Hungarian practitioners of the pastel technique must be mentioned István Nagy, the lowland master, whose heavy pastel pictures are characterized by their dramatic effects and, from the other end of the emotional scale, József Egry, whose sunny Lake Balaton pastels illustrate the sensitive harmony interpreting the lightness of the medium.

By means of the pastel very fine transitions and fade-outs can be illustrated, either in the combined displaying of the visible or non-visible forms. Thus, this technique is perfectly suiting to the illustration of glass and its reflection, having so great an importance on modern architecture.

\subsection{Coloured Prints}

A favoured technique of the modern fine arts - originating primarily from the pop-arts - is the (re)colouring of prints. The series illustrating Andy Warhol, M. Monroe, or E. Presley, painted in various colours is nowadays already a classic. Creators of the present day also readily use the artistic device of the "subjectivized technique".

Naturally, the procedure can also be applied to architectural graphics, since the black and white printed drawings provided by the computer offer a magnificent base for creation of mood and content solutions for such coloured series. Anyone of the above mentioned procedures can be applied to the original surface and in this manner very versatile results and pages of rich graphical appearance are obtained. Expressive spaces of dramatic, sombre mood can be plotted or, by means of the same technique, architectural graphics of a quiet, elegant colourful world can be created.

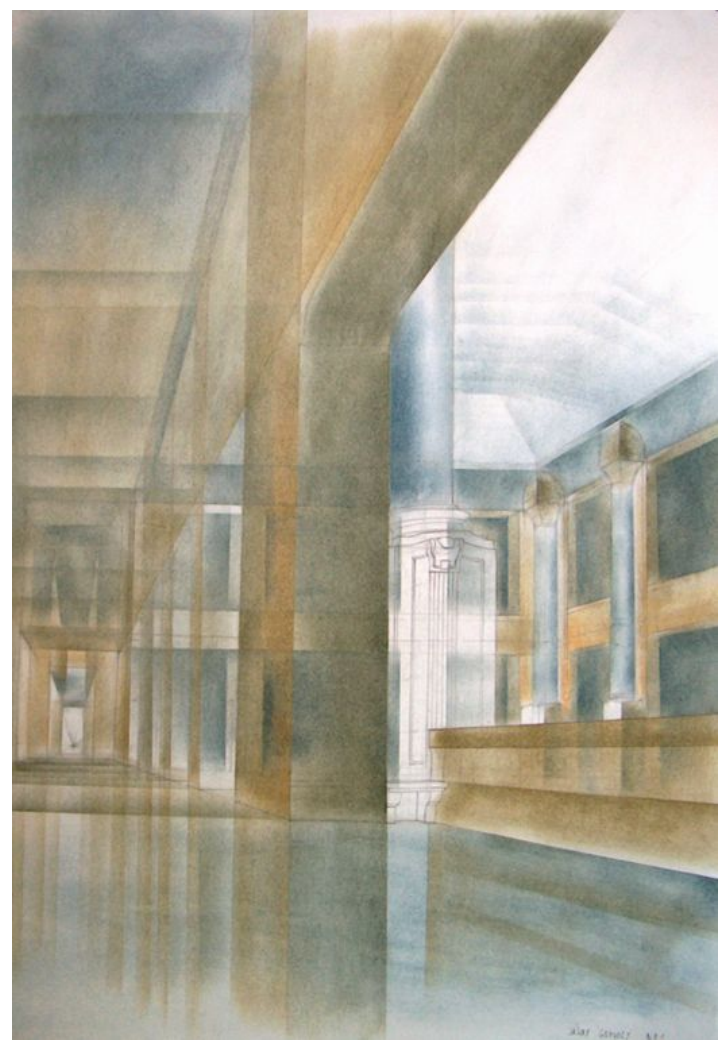

Fig. 5. Central building, student work (G. Jaszai)

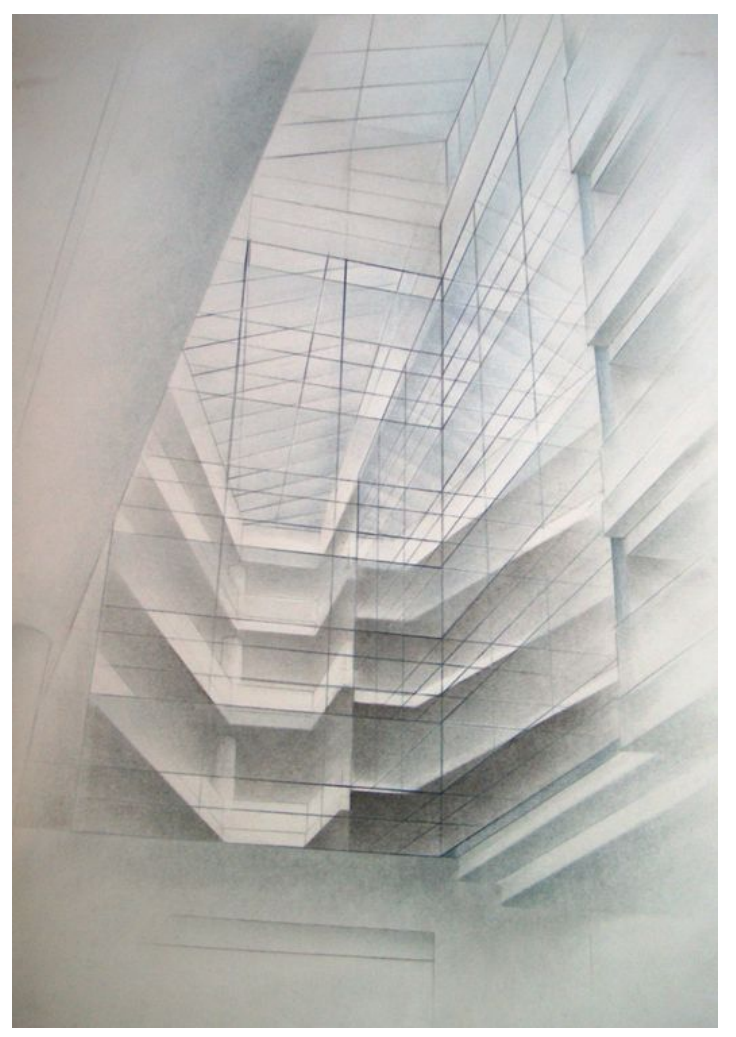

Fig. 6. Info building, student work (Cs. Buella)

\subsection{Abstract Flat Compositions - The Collage}

According to Jonescu: "Since Klee, Kandinszky, Mondrian, Braque and Picasso the art of painting does nothing else than try to liberate itself from anything that is not painting: from literature, the anecdote, history and the photo."

The above citation exactly describes the objectives of the ab- 
stract arts. This aims to create the visual experience, the pictorial representation saturated with aesthetic content, merely with forms and colours, proportions and the harmony thereof. One of the most important trends of the modern arts to the present day is the so-called geometric abstract painting which, starting from the cubist world of thoughts, wants to reach the above outlined objectives through graphic representation of the geometric formations of lines, regular surfaces and forms.

The architectural graphics - mainly the plan graphics, the graphics of plane projections - is tightly bound to this trend of the modern arts, since it also works with these picture elements and its aesthetic content depends on the format, proportion and colour conditions of these picture elements.

The architectural plan graphics can be rightly considered as a special area, a variation of the geometric abstract arts, since "Abstract is every composition which provides nothing in addition to the composition and the elements of the pure colours. The picture itself is the content and the statement of the composition." (Michel Seupor)

Continuing further along this thought it is not an exaggeration to state that a good architect must also have painter's virtues which are indispensable for the creation of the qualities of the building appreciable as an abstract plane composition, for example the face-work or even a ground plan of elegant composition.

An introduction of the relations of abstract composition principles and architectural planning do not form a part of this study, but at the Drawing and Form Faculty important research works are in already in progress in this direction. It is naturally commonly known that the geometric abstract fine arts and modern architecture are in close relationship with each other since they developed mainly parallel to and interacting on each other in such long-acting artistic workshops of the $20^{\text {th }}$ century as the de Stijl movement, or the Bauhaus school of Dessau.

As previously mentioned, the geometric abstract art derives from Cubism - from the so-called "synthetic" summarizing age thereof - which style trend employed the peculiar device of the expression, the collage technique, creating an independent image world from assembling, pasting together different materials and surface structures (from here originates the name: coller(Fr.) $=$ to paste).

The collage technique can be effectively applied to both the plan graphics and to the sight plan representation.

One of the early and fine examples of the sight plan collage is represented by an interior drawing of L. Mies van der Rohe, where the sensitive line drawing is completed to form a composite picture by the photo-realistic, glued elements. Using a simple technique, compositions of serious aesthetic value can be created from the strongly abstracting, abstract drawing and the counterpoint of the exact, detailed reality, by means of the collage.

Another wide area of the utilization of the collage as architectural graphics is the plan graphic.

Magnificent, material-like collages can be created with the

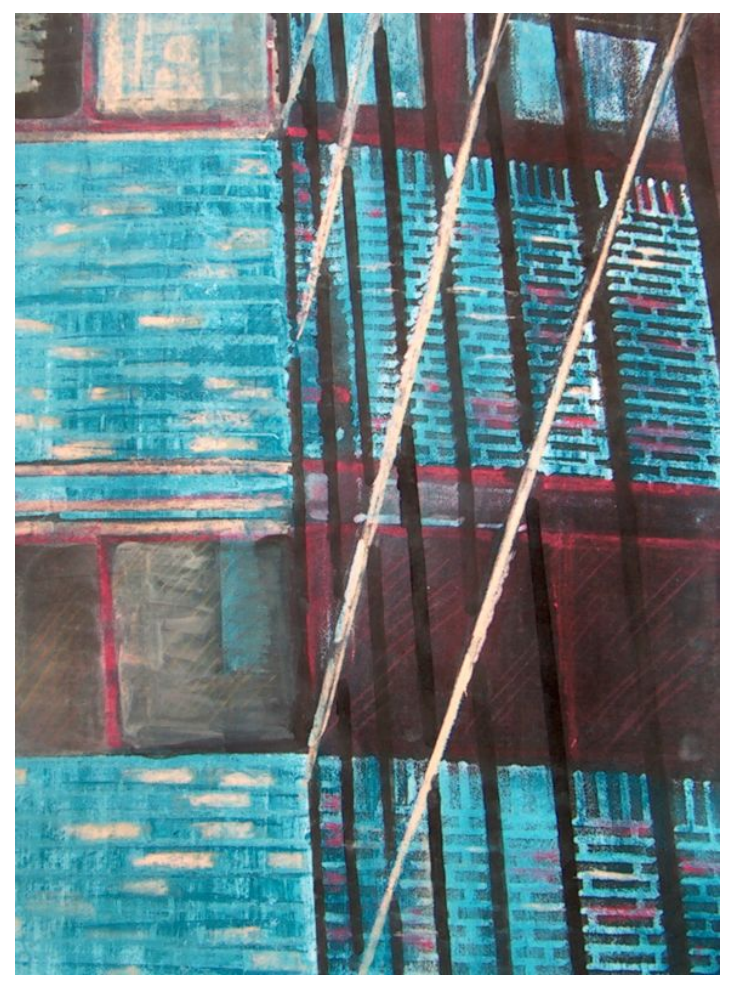

Fig. 7. Info building, student work

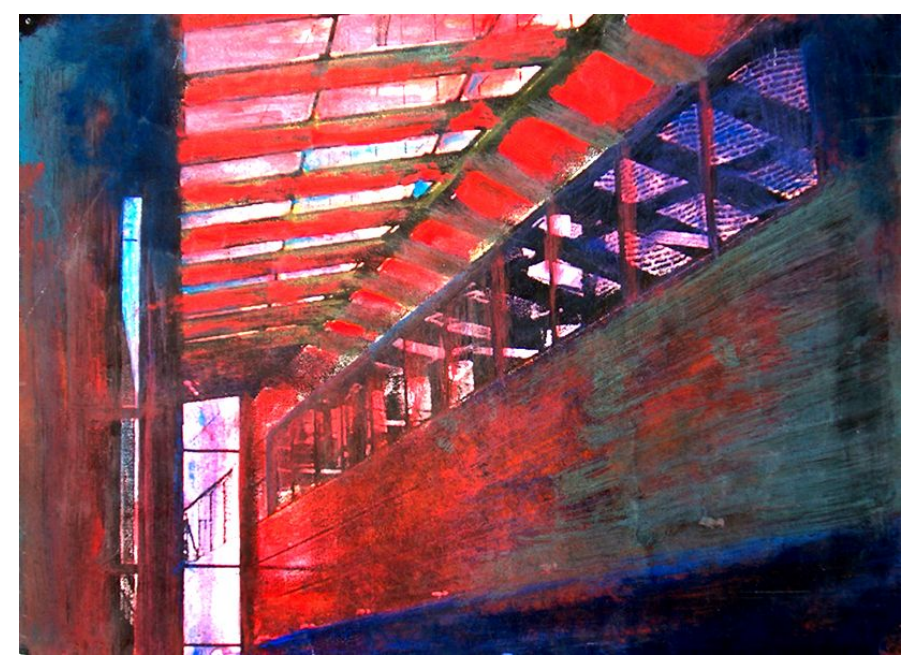

Fig. 8. Info building, student work

use of wood, metal or possibly glass. Collages of this kind consisting of surfaces laminated on each other are already capable of stimulating the illusion of the spatial, three-dimensional representation, the aesthetics of the relief. This technique makes the spatial arrangement of the individual architectural elements easily perceptible, whereby more and more commonly understandable information can be transferred about the building designed.

A further advantage of the collage is that it can be quickly produced and it results in an elegant surface appearance (facilitating a further graphical solution but this is by no means necessary), thus it can be superbly employed as the graphics of design competitions and architectural exhibitions, furthermore, it is an excellent device for the implementation of the individual ideas, offering a wide assortment of materials to be used to meet 
the requirements of individual representation mentioned in the preamble.

As an example, let us look at a collage of the famous American architect Michael Graves and, a few from students' works created at our Faculty, where the three-dimensional character of the original work fades only slightly through the flatness of the reproduction.

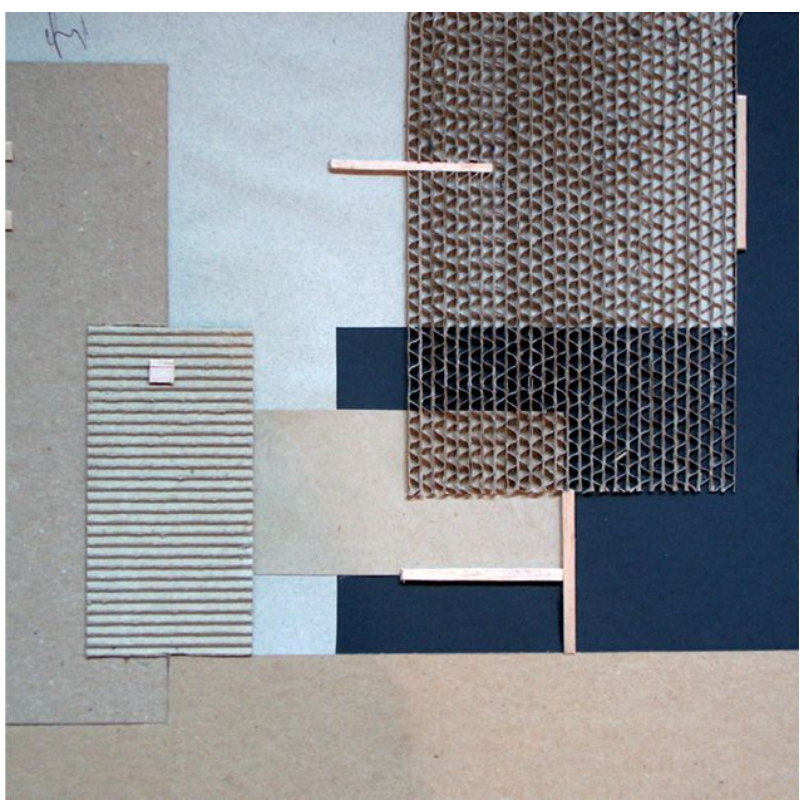

Fig. 9. Floor plan collage, student work (M. Toth)

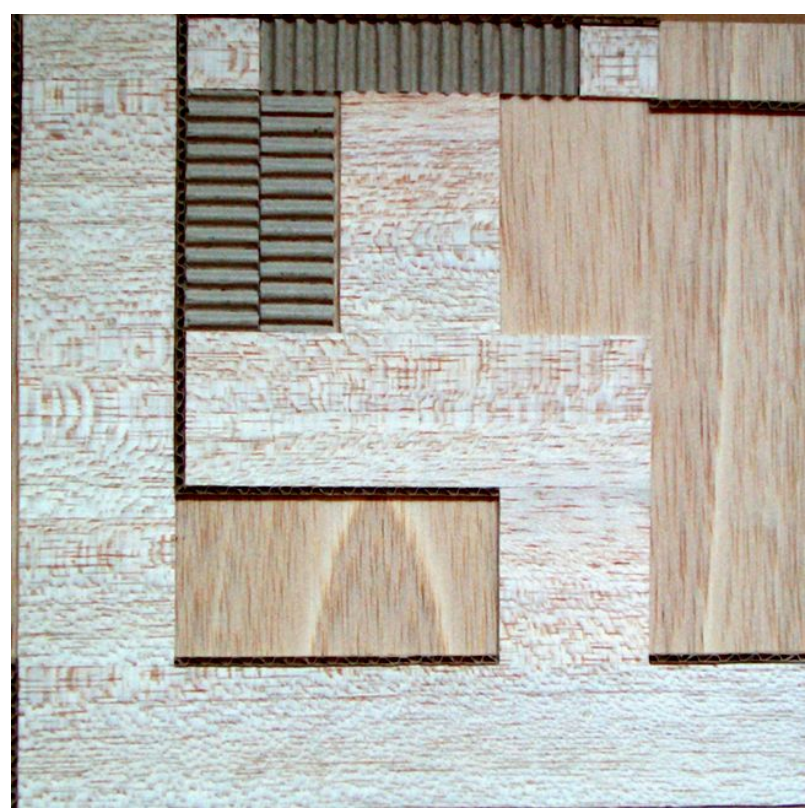

Fig. 10. Floor plan collage, student work (P. Asztalics)

Thus by and large the procedures and drawing techniques available from the history of fine arts for architectural graphics have been discussed. The procedures merely by themselves provide a facility for the creation of very individual and personal graphics and naturally when they are alloyed with each other the possibilities of the individual works are further expanded and, limits are drawn only by the skill, graphic sensitivity and fantasy of the creator, designer or architect. At our Faculty we strive to give all possible help and not least stimulation for future architects to become familiar with these techniques and capable of using them as basic elements of their set of representation devices in the creation of compositions of independent graphic value.

Should the reader by chance miss the discussion of computer graphics in this study, it is done consciously due to the following reasons:

Of the computer graphics two sharply deviating types are discriminated by me: the computer generated drawing and the computer-aided graphic. On computer generated drawing those works are produced where after entering of the required data the computer, on basis of adequate programs, prepares the plan documentation. The sight of either hyper-realistic accuracy created in this manner, which although provides a great help in the introduction of the building to non-professionals, may not be considered as an artistic product because the contribution of the creative person, the "personality" of the graphics - forming one of the major criteria of the artistic creation - is just very limited.

Contrary to this, the computer-aided drawing - where the computer is "only" a drawing device in the tool set of the creator - may result in compositions having already considerable value. However, the artistic tool set of the graphics produced in this manner is broadly identical to the graphic procedures already discussed in this study, furthermore, just the computer (and its programs) as a drawing tool - due to their still rather high technical complexity - requires considerable preliminary training in this field. At the Drawing Faculty naturally we are dealing at a high level with teaching the utilization of the computer - as a creative drawing tool - within the framework of the subjects covering this area.

This study is intended primarily to discuss the graphic techniques of the fine arts, their suggestivity and graphic applicability however, drawing to the end of the study I want also to explain a few thoughts on the theme of "architectural graphics the style of graphics".

Knowledge of the fine arts may provide help not only in the techniques, but in the way of individual presentation respectively in the creation of the graphic way of expression also strengthening the architectural concept. In the recent world of different architectural styles living together it is fortunate if the graphic representation prepared on the planned building and the inspiration of the building are in harmony. The architectural intention and proposition become evident already in the phase of the graphic representation for the customer and the professional public alike.

In this respect the architectural graphics unambiguously referring to the style eras may provide a great help.

But even if the conformity will not be so unambiguous, the stylistics borrowed from the fine arts can always be an enhancing factor to the aesthetic content of the architectural graphics. With a few examples I will try to illustrate my thoughts:

The gaunt, puritan line drawing is a perfect conveyor of the 
pure simplicity of a neo-modern architectural world.

Let us see, how Aldo Rossi utilizes the Chirico's image world on the perspective picture created for his Modena's cemetery tender, undertaking the common emotional and spiritual content; the spiritual unity of the surrealist architectural composition and the mysterious neo-classic world dreamt by the painter decades earlier.

On the perspective drawing prepared by Helmuth Jahn for a bank building in Houston one may accurately feel the effect of the urbane aesthetics of the art-deco, also the visual effect perfectly expressing the idea of "urban life" on the creation titled Radiator Building created by G. O'Keefe in 1927.

These few examples are perhaps sufficient to indicate how illustrious creators of individual ages of the fine arts can contribute to development of the image world of the architectural graphics, although a detailed explanation of this theme forms the subject of a scheduled future study.

However, the first step for the development of an individual, personal architectural graphics style is to familiarize ourselves with the fundamental techniques. This associated with adequate practice, on which basis a drawing style of individual and artistic quality, suiting the approach and architectural style of the designer architect, characteristic as a business card and trademark maybe born.

Postscript:

The presented student's drawings have been created on the author's "Artistic Drawing" and "Architectural Representation" courses. 